\title{
Outcomes of palliative bowel surgery for malignant bowel obstruction in patients with gynecological malignancy
}

\author{
TOMOKO GOTO, MASASHI TAKANO, TADASHI AOYAMA, MORIKAZU MIYAMOTO, AKIO WATANABE, \\ MASAFUMI KATO, NAOKI SASAKI, JUNKO HIRATA, HIDENORI SASA and KENICHI FURUYA
}

Department of Obstetrics and Gynecology, National Defense Medical College, Saitama 359-8513, Japan

Received March 16, 2012; Accepted June 26, 2012

DOI: $10.3892 / 01.2012 .835$

\begin{abstract}
The aim of this study was to evaluate the outcomes of patients with a gynecological malignancy who received palliative care with and without surgical procedures for malignant bowel obstruction (MBO) and to explore prognostic factors to aid the selection of patients who would benefit from palliative surgery. Medical records of patients with MBO due to a gynecological malignancy treated at our institute between 2005 and 2010 were reviewed. Successful palliation following surgery was defined as the ability to tolerate solid food for at least 60 days. Clinical variables were analyzed using Chi-square or Fisher's exact tests. Survival was evaluated using the Kaplan-Meier method and log-rank test. A total of 53 cases were identified; 20 had bowel surgery for MBO as a palliative procedure and 33 did not. Colostomy was performed in $11(55 \%)$ of 20 patients and ileostomy was performed in 7 (35\%). The postoperative morbidity was 35\% and mortality within 30 days was 5\%. Successful palliation following surgery was achieved in $14(70 \%)$ of 20 cases with a median period of 146 days (range, 61-294). Survival following the diagnosis of $\mathrm{MBO}$ was longer in cases with surgery than those without (median survival time, 146 versus 69 days; $\mathrm{P}<0.0001$ ). Although age, presence of ascites, laboratory values and types of prior anticancer therapy were not significantly different, a longer interval from last anticancer therapy to diagnosis of MBO was observed in patients who underwent surgery compared with those who did not (median, 57 versus 30 days; $\mathrm{P}<0.05$ ), as well as superior performance status. Among the patients with surgery, the interval was also longer in patients with successful palliation compared with those without (median, 83 versus 32 days; $\mathrm{P}<0.05$ ). The palliative benefit of surgery for $\mathrm{MBO}$ in selected patients with gynecological malignancy was observed. The interval from last anticancer
\end{abstract}

Correspondence to: Dr Tomoko Goto, Department of Obstetrics and Gynecology, National Defense Medical College, 3-2 Namiki, Tokorozawa, Saitama 359-8513, Japan

E-mail: tgoto@ndmc.ac.jp

Key words: malignant bowel obstruction, gynecological malignancy, palliative care, surgery, prognosis, treatment-free interval therapy to diagnosis of $\mathrm{MBO}$ may serve as a prognostic factor when considering surgical intervention.

\section{Introduction}

Gynecological malignancy may develop into bowel obstruction at primary presentation, but obstruction occurs more frequently with recurrence and disease progression as the consequence of resistance to and failure of anticancer therapy. This often results in poor quality of life and prolonged hospitalization at the end of life. Several prognostic factors for malignant bowel obstruction (MBO) have been reported to date, including age, performance status, nutritional status, ascites, palpable mass, extraabdominal metastasis and previous anticancer treatment (1-5). Although there have been increased efforts in the integration of palliative care, clinicians are in need of clinical data to guide towards more evidence-based practice. A number of previous studies have investigated the effects and outcomes of palliative surgery for MBO; however, the role of surgical treatment for terminal patients is controversial (6). Differences in outcome measures, including symptom control, quality of life and survival, without the establishment of a standardized assessment, makes it difficult to interpret each result, leading to further confusion. Moreover, patients who did not have surgery are usually not analyzed in the same way and there are few data which have been investigated by prospective study or multivariate analysis, despite the frequent occurrence of MBO in patients with gynecological malignancy; consequently, most previous studies have been unable to define criteria that would allow the selection of patients who are unlikely to benefit from surgery $(7,8)$. The purpose of this study was to evaluate the outcomes of patients with gynecological malignancy who received palliative care with and without surgical procedure for MBO in our institute and to explore prognostic factors to aid the selection of patients who would benefit from palliative surgery.

\section{Patients and methods}

Patients. Of all the patients with gynecological malignancy treated at our institute, medical records of patients who presented with $\mathrm{MBO}$ due to disease progression or recurrence between 2005 and 2010 were reviewed. Cases with symptoms of bowel obstruction which were temporary and restorable 
with short medical treatment were excluded. All patients underwent a physical examination, a plain film and a CT scan evaluation. Nasogastric or long intestinal tubes for decompression and symptom relief were frequently attempted during the course of initial conservative treatment. Water-soluble contrast medium was also administered via tubes when considered appropriate to detect sites of strangulation. The study protocol was approved by the ethics committee of the National Defense Medical College, Saitama, Japan. Patient consent was obtained either from the patient or the patient's family

Surgical procedures. Bowel surgery to palliate MBO was considered only for patients with relatively good performance status, predicted life expectancy longer than 60 days, expectation of surgically possible decompression by image scan or contrast radiography leading to resumption of oral diet and strong desire for surgery, including stoma formation. Laparoscopy was not performed during the study period. Patients whose obstruction was present at diagnosis of primary disease and corrected at primary surgery were excluded. Bowel surgery to release bowel obstruction performed together with cytoreductive surgery was not defined as palliative surgery and was also excluded from the present study.

Outcomes. Successful palliation following surgery was defined as the ability to tolerate solid food for at least 60 days, as defined in several previous studies $(4,9)$. The treatment-free interval was calculated from the time when prior treatment was completed to the time of diagnosis for MBO, which was defined as the first time that bowel obstruction was diagnosed by radiographic or physical examination following gastrointestinal symptoms due to obstruction. Overall survival was measured from the date of diagnosis for $\mathrm{MBO}$ to the date of mortality or last follow-up.

Statistical analysis. Associations between clinical variables were analyzed using Chi-square or Fisher's exact tests. Survival curves were estimated using the Kaplan-Meier method and $\mathrm{P}$-values were generated using the log-rank test. $\mathrm{P}<0.05$ was considered to indicate a statistically significant difference.

\section{Results}

Characteristics of the patients. During the study period, 53 patients who required hospitalization and palliative care for MBO at our institute were identified. Table I provides a summary of patient characteristics at diagnosis of MBO. The ovary was the most common primary site and accounted for $>60 \%$ of the cohort, including Fallopian tube and primary peritoneum. Approximately half of the cases (25/53) had massive ascites diagnosed by image scan. Of all the cases, $20(38 \%)$ had bowel surgery for MBO as a palliative procedure (operative group) and 33 (62\%) did not (non-operative group).

Surgical procedures and outcomes. Table II describes the palliative surgical procedures performed for $\mathrm{MBO}$ in the operative group and their outcomes. Colostomy was performed in $11(55 \%)$ of 20 patients and ileostomy was in $7(35 \%)$ patients. Postoperative complications were observed in 7 (35\%) of the 20 patients. Of the complications, infections, including wound
Table I. Characteristics of patients with palliative care for MBO due to gynecological malignancy $(\mathrm{n}=53)$.

\begin{tabular}{lc}
\hline Characteristic & \multicolumn{1}{c}{ Value } \\
\hline Age, median (range), years & $58(35-85)$ \\
Origin of primary disease, $\mathrm{n}(\%)$ & \\
Cervix & $9(17)$ \\
Corpus & $11(21)$ \\
Ovary & $29(55)$ \\
Fallopian tube & $1(2)$ \\
Primary peritoneal & $3(6)$ \\
ECOG performance status, $\mathrm{n}(\%)$ & \\
1 & $18(34)$ \\
2 & $23(43)$ \\
3 & $11(21)$ \\
4 & $1(2)$
\end{tabular}

History of anticancer therapy ${ }^{\mathrm{a}}, \mathrm{n}(\%)$

Surgery

Chemotherapy

Radiotherapy

Time from primary therapy to diagnosis of $\mathrm{MBO}$, median (range), days

$780(30-4486)$

Treatment-free interval between last anticancer therapy and diagnosis of MBO, median (range), days

Presence of massive ascites, $\mathrm{n}(\%)$

Yes

No

Palliative procedure performed for MBO, n (\%)

Pharmacological treatment only

Laparotomy

$20(38)$

ECOG, Eastern Cooperative Oncology Group. ancludes more than one per patient. MBO, malignant bowel obstruction.

dehiscence, abscess formation and sepsis, were relatively frequent and successfully resolved by antibiotic treatment. Although no patients underwent open-and-shut laparotomy only without any corrective surgery taking place, $2(11 \%)$ patients' symptoms were not relieved without the resumption of oral diet by surgical correction and 1 patient tolerated an oral diet following surgery but succumbed to disease progression within a month. Successful palliation, as defined in Patients and methods, was achieved in 14 (70\%) of the 20 cases, with a median period of 146 days (range, 61-294). The median survival time following surgery was 143 days (range, 24-294) and after diagnosis of MBO was 146 days (range, 40-334).

Survival analysis. The Kaplan-Meier test for survival analysis (Fig. 1) confirmed significantly longer survival following the diagnosis of $\mathrm{MBO}$ in the operative than the non-operative group (median survival time, 146 versus 69 days; $\mathrm{P}<0.0001$ ). Comparisons of characteristics between the two groups are shown in Table III. As expected, the performance status at 
Table II. Palliative surgical procedures performed for $\mathrm{MBO}$ and outcomes $(n=20)$.

\begin{tabular}{|c|c|}
\hline Characteristic & Value \\
\hline Surgical procedure performed ${ }^{\mathrm{a}}, \mathrm{n}$ & 20 \\
\hline Transverse colostomy & 10 \\
\hline Descending colostomy & 1 \\
\hline Ileostomy & 7 \\
\hline Bypass & 7 \\
\hline $\begin{array}{l}\text { Time from diagnosis of MBO to surgery, } \\
\text { median (range), days }\end{array}$ & $9(1-63)$ \\
\hline Operating time, median (range), minutes & $97(32-257)$ \\
\hline Blood loss, median (range), gram & $97(5-4620)$ \\
\hline $\begin{array}{l}\text { Time to resume diet following surgery, } \\
\text { median (range), days }\end{array}$ & $5(2-23)$ \\
\hline Postoperative complications ${ }^{\mathrm{a}}, \mathrm{n}(\%)$ & $7(35)$ \\
\hline Abscess & 4 \\
\hline Wound infection and dehiscence & 3 \\
\hline Sepsis & 1 \\
\hline Deep venous thrombosis & 1 \\
\hline Short bowel syndrome & 1 \\
\hline Symptoms unrelieved, n (\%) & $2(10)$ \\
\hline Postoperative mortality within 30 days, n (\%) & $1(5)$ \\
\hline $\begin{array}{l}\text { Duration capable of postoperative } \\
\text { oral diet, median (range), days }\end{array}$ & $100(0-294)$ \\
\hline $\begin{array}{l}\text { Successful palliation following surgery } \\
\text { (>60 days), n }(\%)\end{array}$ & $14(70)$ \\
\hline On discharge taking oral diet, $\mathrm{n}(\%)$ & $15(75)$ \\
\hline $\begin{array}{l}\text { Median survival time following surgery } \\
\text { (range), days }\end{array}$ & $143(24-294)$ \\
\hline $\begin{array}{l}\text { Median survival time following diagnosis } \\
\text { of MBO (range), days }\end{array}$ & $146(40-334)$ \\
\hline
\end{tabular}

Includes more than one per patient. MBO, malignant bowel obstruction.

diagnosis of MBO was superior in the operative group. Other variables used to assess potential medical conditions, including age, presence of massive ascites and laboratory values, were not significantly different between the groups; however, a longer interval from the last anticancer therapy to diagnosis of MBO was observed in the operative than the non-operative group (median, 57 versus 30 days; $\mathrm{P}<0.05$ ). Further analysis comparing the details of the previous anticancer treatment revealed that there was no significant difference in the number of chemotherapeutic regimens or courses performed, the number of previous laparotomy or bowel surgeries performed or previous radiotherapy of the abdomen or pelvis. Three patients had stoma at the onset of MBO in the non-operative group and 1 in the operative group (not statistically significant). Based on these results, we performed a further comparative analysis of the characteristics of the operative group (Table IV), comparing patients who achieved successful

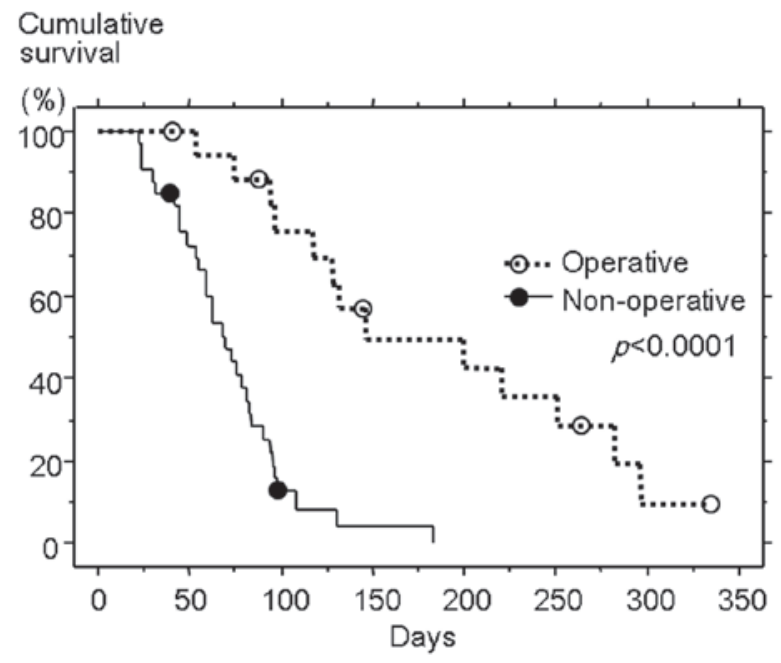

Figure 1. Comparison of overall survival following the diagnosis of malignant bowel obstruction between the operative and non-operative groups. The operative group had significantly longer survival than the non-operative group $(\mathrm{P}<0.0001)$.

palliation following surgery (successful group; $n=14$ ) with those who did not (unsuccessful group; $n=6$ ). The interval from the last anticancer therapy to diagnosis of $\mathrm{MBO}$ was also longer in the successful than the unsuccessful group (median, 83 versus 32 days; $\mathrm{P}<0.05$ ), although the performance status did not show a marked difference. Time from diagnosis of MBO to surgery, site of stoma and postoperative complications also had no impact on surgical outcome according to the comparison of these two groups.

\section{Discussion}

In our cases of MBO due to gynecological malignancy, the most predominant primary disease was ovarian cancer, accounting for more than half of the cases. Reflecting the large population, approximately half of the cases suffered from massive ascites at the onset of MBO. Ovarian cancers frequently present with intraabdominal dissemination without impairment of vital organs in the course of disease progression and recurrence, leading to peritonitis carcinomatosa with impaired bowel movements. Successful palliation following surgery was observed in our patients with ovarian cancer who presented with ascites requiring occasional paracentesis. Considering that neither the proportion of patients with ovarian cancer nor those with ascites was significantly different in the two comparisons of operative versus non-operative group and successful versus unsuccessful group, such patients could not be excluded from the candidacy for palliative surgery and careful assessment should be individually required.

In the present study, significantly longer survival following the diagnosis of $\mathrm{MBO}$ in the operative than the non-operative group was observed, in accordance with several previous studies $(3,10,11)$. By comparing the characteristics of the two groups, the performance status at diagnosis of $\mathrm{MBO}$ was found to be superior in the operative group as expected, since surgery had been considered only for patients with a relatively good performance status, unsurprisingly leading to the longer survival in the operative group. In addition to 
Table III. Comparison of characteristics of patients between the operative $(n=20)$ and non-operative groups $(n=33)$.

\begin{tabular}{|c|c|c|c|}
\hline Characteristics & Operative $(\mathrm{n}=20)$ & Non-operative $(\mathrm{n}=33)$ & P-value \\
\hline Age, median (range), years & $58(35-84)$ & $59(40-85)$ & N.S. \\
\hline Primary site, $\mathrm{n}(\%)$ & & & N.S. \\
\hline Cervix & $2(10)$ & $7(21)$ & \\
\hline Corpus & $6(30)$ & $5(15)$ & \\
\hline Ovary & $12(60)$ & $17(52)$ & \\
\hline Fallopian tube & 0 & $1(3)$ & \\
\hline Peritoneum & 0 & $3(9)$ & \\
\hline ECOG performance status, $\mathrm{n}(\%)$ & & & $<0.001$ \\
\hline$<2$ & $15(75)$ & $3(9)$ & \\
\hline$\geq 2$ & $5(25)$ & $30(91)$ & \\
\hline History of anticancer therapy ${ }^{\mathrm{a}}, \mathrm{n}(\%)$ & & & N.S. \\
\hline Surgery & $19(95)$ & $31(94)$ & \\
\hline Chemotherapy & $19(95)$ & $31(94)$ & \\
\hline Radiotherapy & $7(35)$ & $10(30)$ & \\
\hline $\begin{array}{l}\text { Time from primary therapy to diagnosis } \\
\text { of MBO, median (range), days }\end{array}$ & $820(43-4486)$ & $720(30-2430)$ & N.S. \\
\hline $\begin{array}{l}\text { Treatment-free interval between last anticancer } \\
\text { therapy and diagnosis of MBO, median (range), days }\end{array}$ & $57(6-512)$ & $30(8-360)$ & $<0.05$ \\
\hline Presence of massive ascites, $\mathrm{n}(\%)$ & & & N.S. \\
\hline Yes & $8(40)$ & $17(52)$ & \\
\hline No & $12(60)$ & $16(48)$ & \\
\hline Laboratory values, median (range) & & & N.S. \\
\hline Hemoglobin, g/dl & $10.0(6.7-13.0)$ & $9.3(6.3-14.0)$ & \\
\hline Serum albumin, g/dl & $2.8(1.8-3.6)$ & $2.9(1.6-4.3)$ & \\
\hline White blood cell count (WBC), $/ \mu 1$ & $5400(2790-21390)$ & $5300(1410-16220)$ & \\
\hline Total lymphocyte count (TLC), $/ \mu 1$ & $767(362-2195)$ & 705 (106-2201) & \\
\hline C-reactive protein (CRP), mg/dl & $5.3(0.1-23.7)$ & $3.4(0.3-27.3)$ & \\
\hline
\end{tabular}

ECOG, Eastern Cooperative Oncology Group; MBO, malignant bowel obstruction; N.S., not significant. ancludes more than one per patient.

performance status, a longer interval from last anticancer therapy to diagnosis of MBO was observed in the operative than the non-operative group. In our comparisons in the operative group, the successful group also showed a longer interval from the last anticancer therapy to the diagnosis of MBO than the unsuccessful group, despite no marked difference in performance status. However, we did not find any difference in the type nor the number of anticancer therapy. Since our patients did not receive further chemotherapy following the diagnosis of $\mathrm{MBO}$, the implication should be different from that of the treatment-free interval as a good prognostic factor to predict a better response to further anticancer therapy in recurrent ovarian cancer; however, patients with a longer treatment-free interval despite having progressive disease or recurrence may have a superior nutritional status, be relatively stable in their disease and be better prepared for palliative therapy, possibly resulting in a good outcome. Zoetmulder et al also reported that the interval between last treatment and bowel obstruction, followed by the presence of ascites, was the most significant prognostic factor for $\mathrm{MBO}$ in ovarian cancer, discussing the implication of a long interval reflecting differences in the biology of relatively slow-growing tumors (3). A short interval between the diagnosis of primary cancer and bowel obstruction correlated with lower survival probabilities, according to Fernandes et al (2). In our cases, the time from primary therapy to diagnosis of $\mathrm{MBO}$ was longer in the operative and successful groups when compared with their opponents, but was not statistically significant, similar to the result reported by Zoetmulder et al (3).

Concerning previous anticancer therapy, there was no significant difference in the history of previous bowel surgery when operative versus non-operative and successful versus unsuccessful group comparisons were performed; however, three patients had stoma at the onset of MBO in the non-operative group, which were constructed at primary or secondary debulking surgery, while there was only one case with stoma, which was constructed due to rectovaginal fistula following recurrence, in the operative group. Pothuri et al reported their experiences of reoperation for palliation of recurrent $\mathrm{MBO}$ in 
Table IV. Comparison of characteristics of patients between successful $(n=14)$ and unsuccessful ( $n=6)$ palliation groups following surgery for $\mathrm{MBO}$.

\begin{tabular}{|c|c|c|c|}
\hline Characteristics & Successful $(n=14)$ & Unsuccessful (n=6) & P-value \\
\hline Age, median (range), years & $59(35-68)$ & $54(45-84)$ & N.S. \\
\hline Primary site, $\mathrm{n}(\%)$ & & & N.S. \\
\hline Cervix & $2(14)$ & $0(0)$ & \\
\hline Corpus & $5(36)$ & $1(17)$ & \\
\hline Ovary & $7(50)$ & $5(83)$ & \\
\hline ECOG performance status, n (\%) & & & N.S. \\
\hline$<2$ & $11(79)$ & $4(67)$ & \\
\hline$\geq 2$ & $3(21)$ & $2(33)$ & \\
\hline History of anticancer therapy ${ }^{\mathrm{a}}, \mathrm{n}(\%)$ & & & N.S. \\
\hline Surgery & $13(93)$ & $6(100)$ & \\
\hline Chemotherapy & $13(93)$ & $6(100)$ & \\
\hline Radiotherapy & $6(43)$ & $1(17)$ & \\
\hline $\begin{array}{l}\text { Time from primary therapy to diagnosis } \\
\text { of MBO, median (range), days }\end{array}$ & $855(271-4486)$ & $513(43-3768)$ & N.S. \\
\hline $\begin{array}{l}\text { Treatment-free interval between last anticancer } \\
\text { therapy and diagnosis of MBO, median (range), days }\end{array}$ & $83(33-512)$ & $32(6-74)$ & $<0.05$ \\
\hline Presence of massive ascites, n (\%) & & & N.S. \\
\hline Yes & $5(36)$ & $3(50)$ & \\
\hline No & $9(64)$ & $3(50)$ & \\
\hline Laboratory values, median (range) & & & N.S. \\
\hline Hemoglobin, g/dl & $10.3(8.5-13.0)$ & $7.4(6.7-10.9)$ & \\
\hline Serum albumin, g/dl & $2.8(1.8-3.5)$ & $2.8(1.9-3.6)$ & \\
\hline White blood cell count (WBC), / $\mu 1$ & $6100(2790-21390)$ & $5400(5100-7300)$ & \\
\hline Total lymphocyte count (TLC), / $\mu 1$ & $762(362-2195)$ & $759(383-968)$ & \\
\hline C-reactive protein $(\mathrm{CRP}), \mathrm{mg} / \mathrm{dl}$ & $2.5(0.1-23.7)$ & $5.7(4.9-21.0)$ & \\
\hline Time from diagnosis of MBO to surgery, median (range), days & $10(1-40)$ & $6(3-63)$ & N.S. \\
\hline Site of stoma, n (\%) & & & N.S. \\
\hline Colostomy & $7(50)$ & $4(67)$ & \\
\hline Ileostomy & $5(36)$ & $2(33)$ & \\
\hline $\begin{array}{l}\text { Multiple bowel procedures, including bypass } \\
\text { at palliative surgery, } \mathrm{n}(\%)\end{array}$ & $4(29)$ & $1(17)$ & N.S. \\
\hline Postoperative complications, n (\%) & $6(43)$ & $2(33)$ & N.S. \\
\hline Median survival time following surgery (range), days & $192(67-294)$ & $64(24-143)$ & $<0.05$ \\
\hline Median survival time following diagnosis of MBO (range), days & $221(75-334)$ & $94(40-146)$ & $<0.05$ \\
\hline
\end{tabular}

ECOG, Eastern Cooperative Oncology Group; N.S., not significant. ${ }^{a}$ Includes more than one per patient. MBO, malignant bowel obstruction.

ovarian cancer and concluded that patients undergoing repeat surgery for recurrent bowel obstruction have a low likelihood of successful palliation, although the details of their previous palliative surgeries were not described (9). Considering their results, patients who had a history of bowel surgery to correct bowel obstruction, especially stoma, may not be suitable even for the first palliative bowel surgery, as a poor possibility of surgical correction and high morbidity rate were expected.

Also concerning previous anticancer therapy, approximately half of our patients (6/14) with successful palliation had prior radiotherapy (for 2 cervical, 2 corpus and 2 ovarian cancers), compared with 1 of the 6 patients in the unsuccessful group. Conflicting results have been reported concerning the prognostic significance of prior radiotherapy on MBO. Krebs and Goplerud reported that previous radiotherapy of the abdomen was a poor prognostic indicator of low likelihood of clinical benefit from surgery to treat MBO (1). They explained that patients treated by radiotherapy were found to be inoperable at laparotomy or developed severe postoperative bowel complications, including dehiscence of bowel anastomosis and formation of enterocutaneous fistulas. Postoperative complications were observed in 3 of our 7 patients with prior 
radiotherapy, including 2 infections and 1 case of short bowel syndrome. By contrast, Fernandes et al reported that a lack of previous radiotherapy resulted in lower survival probabilities in patients with ovarian cancer and bowel obstruction (2). Although multiple factors associated with radiotherapy may affect the outcomes of patients with MBO, 6 (86\%) of our 7 patients with prior radiotherapy had successful palliation following surgery; therefore, irradiated patients may not always constitute an unfavorable subgroup of patients when considering surgical intervention.

Time from diagnosis of MBO to surgery, site of stoma and postoperative complications had no impact on surgical outcome according to the comparison of the successful and unsuccessful groups. The reason why postoperative morbidity did not correlate with successful palliation following surgery was probably due to the complications observed in our cases recovering with appropriate medications. A wide range of morbidity and mortality rates following surgery for $\mathrm{MBO}$ have been reported in the previous studies, varying from 5 to $49 \%$ for the morbidity rate $(4,12)$. Careful perioperative management as well as deliberate selection of patients is important to achieve the palliative goal in these terminally ill patients. The weakness of this study is that the small number of patients analyzed may preclude the detection of statistically significant results, and further clinical data should be gathered by large prospective studies to enable physicians to apply clear criteria for the selection of patients who are likely to benefit from surgery.

In conclusion, our study demonstrated the palliative benefit by bowel surgery for MBO in selected patients with gynecological malignancy; therefore, surgery may be considered as a measure of palliative care for MBO in patients who had a life expectancy of longer than 2 months without serious contraindications and poor performance status. In addition, the interval from last anticancer therapy to diagnosis of MBO may also serve as a prognostic factor when considering surgical intervention.

\section{References}

1. Krebs HB and Goplerud DR: Surgical management of bowel obstruction in advanced ovarian carcinoma. Obstet Gynecol 61: 327-330, 1983

2. Fernandes JR, Seymour RJ and Suissa S: Bowel obstruction in patients with ovarian cancer: a search for prognostic factors. Am J Obstet Gynecol 158: 244-249, 1988.

3. Zoetmulder FA, Helmerhorst TJ, van Coevorden F, Wolfs PE, Leyer JP and Hart AA: Management of bowel obstruction in patients with advanced ovarian cancer. Eur J Cancer 30A: 1625-1628, 1994

4. Ripamonti $\mathrm{C}$ and Bruera E: Palliative management of malignant bowel obstruction. Int J Gynecol Cancer 12: 135-143, 2002.

5. Medina-Franco H, García-Alvarez MN, Ortiz-López LJ and Cuairán JZ: Predictors of adverse surgical outcome in the management of malignant bowel obstruction. Rev Invest Clin 60: 212-216, 2008.

6. Kucukmetin A, Naik R, Galaal K, Bryant A and Dickinson HO: Palliative surgery versus medical management for bowel obstruction in ovarian cancer. Cochrane Database Syst Rev 7: CD007792, 2010.

7. Castaldo TW, Petrilli ES, Ballon SC and Lagasse LD: Intestinal operations in patients with ovarian carcinoma. Am J Obstet Gynecol 139: 80-84, 1981.

8. Rubin SC, Hoskins WJ, Benjamin I and Lewis JL Jr: Palliative surgery for intestinal obstruction in advanced ovarian cancer. Gynecol Oncol 34: 16-19, 1989.

9. Pothuri B, Meyer L, Gerardi M, Barakat RR and Chi DS: Reoperation for palliation of recurrent malignant bowel obstruction in ovarian carcinoma. Gynecol Oncol 95: 193-195, 2004.

10. Mangili G, Aletti G, Frigerio L, Franchi M, Panacci N, Viganò R, DE Marzi P, Zanetto F and Ferrari A: Palliative care for intestinal obstruction in recurrent ovarian cancer: a multivariate analysis. Int J Gynecol Cancer 15: 830-835, 2005.

11. Chi DS, Phaëton R, Miner TJ, Kardos SV, Diaz JP, Leitao MM Jr, Gardner G, Huh J, Tew WP, Konner JA, et al: A prospective outcomes analysis of palliative procedures performed for malignant intestinal obstruction due to recurrent ovarian cancer. Oncologist 14: 835-839, 2009.

12. Feuer DJ, Broadley KE, Shepherd JH and Barton DP: Systematic review of surgery in malignant bowel obstruction in advanced gynecological and gastrointestinal cancer. The Systematic Review Steering Committee. Gynecol Oncol 75: 313-322, 1999. 